\title{
Contribuição ao conhecimento da Flora do Aripuanã (Mato Grosso)
}

\section{Fanerógamas}

\author{
Pedro L. Lisbôa ( ${ }^{*}$ ) \\ Ghillean T. Prance $(* *)$ \\ Regina L. Lisbôa $\left({ }^{*}\right)$
}

\section{Resumo}

Os autores apresentam uma relação de espécies coletadas na região de Aripuanã (Mato Grosso), cuja finalidade é tão somente contribuir para o conhecimento da flora local. Foram incluídas no trabalho somente as plantas em processo de floração e/ou frutificação durante o período de coleta.

\section{INTRODUÇÃo}

A parte sul da floresta amazônica localizada no Estado de Mato Grosso apresenta o mesmo aspecto homogêneo de outras regiões da Amazônia, com ocorrências de floresta tropical de terra firme e floresta de várzea predominantes. Segundo a terminologia de Pires (1973), esta região é também rica em igapós formados pelas áreas baixas, próximas do leito dos rios, e que sofrem alagações periódicas. Durante o abaixamento do nível dessas águas, aparecem extensas praias de areia branca.

Em 1974, o Projeto Radam (Brasil. D.N.P.M. 1974) procedeu a inventários florestais na região de Aripuanã, com a finalidade de avaliar o potencial madeireiro da região e sua possível aplicação. Nesse estudo foram consideradas somente as árvores de interesse comercial por qualidade e volume de madeira. Foram excluídos, assim, não só os indivíduos de porte inferior ao recomendável para explotação bem como as epífitas e trepadeiras superiores e também a flora criptogâmica.

A flora epifítica é bastante diversificada naquela região, sobretudo a brioflora situada na vegetação adjacente ao Salto de Dardanelos (ver foto 1 ), onde a umidade proporciona- da por esta cachoeira favorece o aparecimento de uma flora briofítica exuberante e variada.

Este trabalho visa a contribuir para o conhecimento da flora do Aripuanã no seu todo, ou seja, incluindo plantas de diferentes hábitos e "habitats". O mesmo será composto de duas etapas: nesta, a primeira, estão relacionadas as espécies pertencentes ao grupo das Gymnospermas e Angiospermas. Num segundo trabalho, trataremos da flora briofítica, sendo isto necessário em decorrência da amplitude desse grupo na região do Aripuanã.

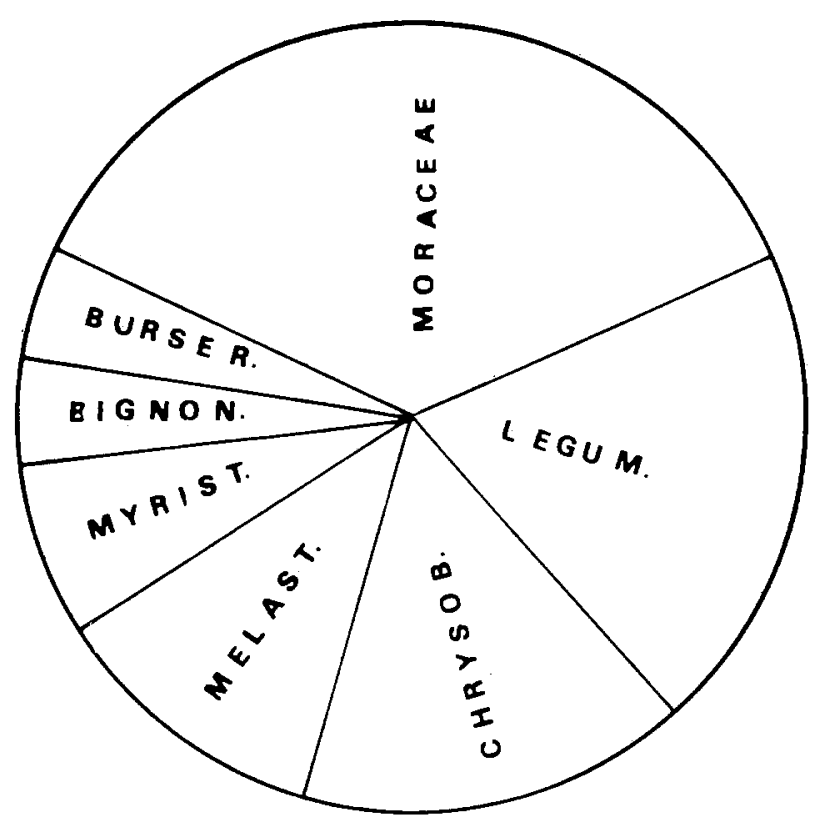

Graf. I - Frequiência de espécies em floração e/ou frutificação por família, coletadas no Município de Aripuanã (MT).

(*) - Instituto Nacional de Pesquisas da Amazônia, Manaus.

(") - New York Botanical Garden. 


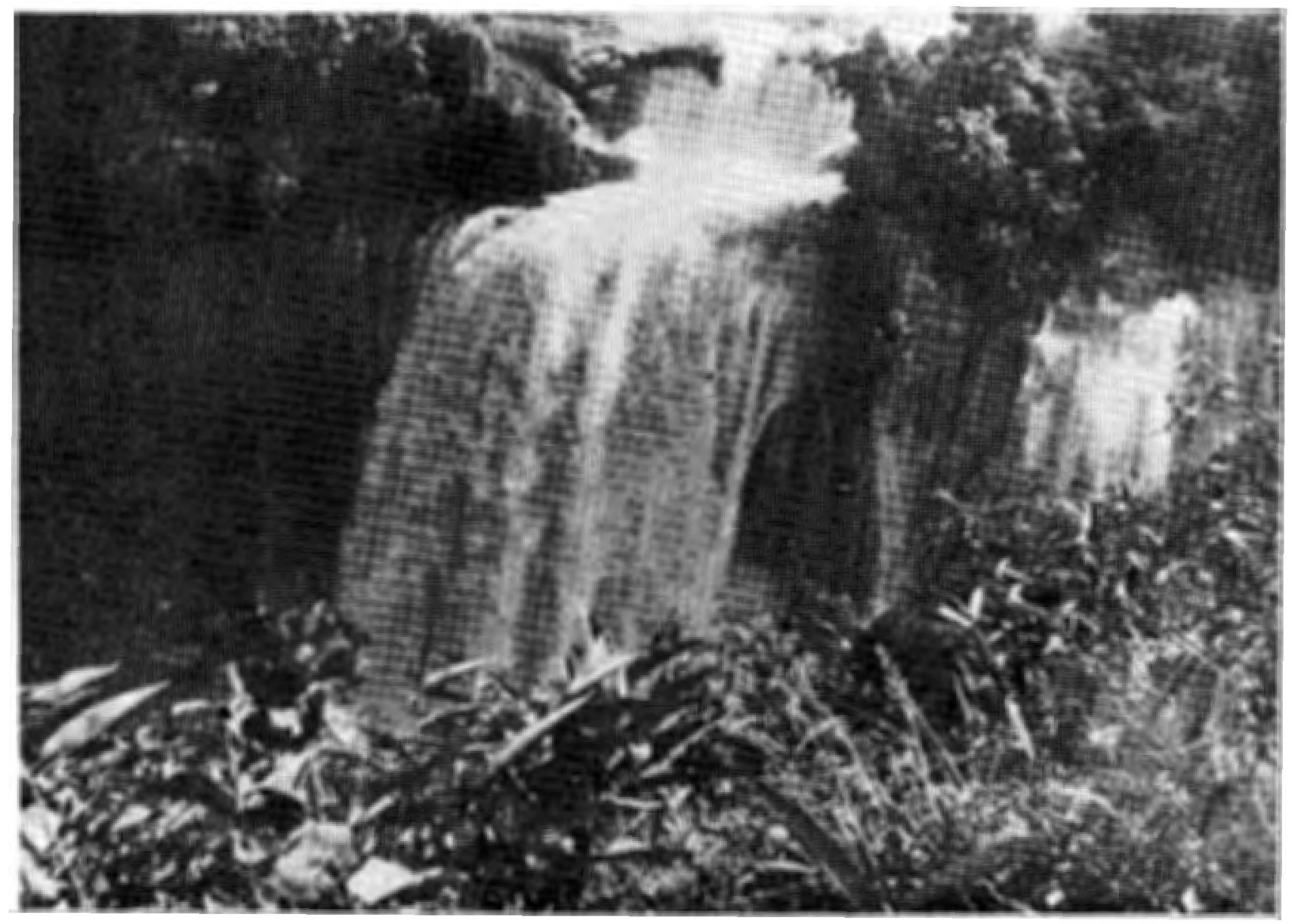

Foto 1 - Vista parcial do Salto de Dardanelos (130m de queda).

Material E MÉTodos

A região oeste da Amazônia Matogrossense é cortada pelos rios Aripuanã e Juruena. $O$ Município de Aripuanã situa-se entre as coordenadas $7^{\circ} 19^{\prime} 45^{\prime \prime}-12^{\circ} 22^{\prime} 30^{\prime \prime}$ de latitude Sul

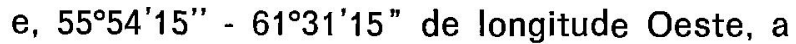
uma altitude que varia de 200 a $300 \mathrm{~m}$ (Brasil. M.P.C.G., Projeto Aripuanã, 1973).

O clima é tropical de altitude e o regime pluviométrico compreende o período de outubro a abril, sendo mais intenso de janeiro a março.

No município, mais precisamente na localidade de Dardanelos, situa-se a estação Humboldt, cuja finalidade é proceder ao desenvolvimento integrado do município; é considerada, no momento, como posto de coleta de material para pesquisas (ver fig. 1).
O material aqui apresentado é relativo a dois períodos de coletas. O primeiro, realizado durante o mês de outubro de 1973 e o outro durante partes dos meses de setembro e outubro de 1975. A coleta consistiu somente de plantas em processo de floração e/ou frutificação, o que dá uma idéia sobre o período de reprodução dessas plantas.

A vegetação do Município de Aripuanã apresenta variações estruturais e de composição em decorrência da variação dos sclos da região. O relatório do Projeto Radam (Brasil. D.N.P.M., 1974) considera dois sistemas florestais na região: um ocupando a plataforma arenítica de Dardanelos e outro que cobre a superfície plana do embasamento granito-gnaisse.

Os locais explorados neste estudo foram os seguintes: mata de terra firme adjacente 
ao aeroporto, picada Humboldt-Vilhena, ambos os locais com solo argiloso; matas de igapós pantanosos ao longo das margens dos rios Aripuanã e Juruena e matas de igapós arenosos na cachoeira dos Patos e Salto de Dardanelos.

Após a discussão e conclusões, é apresentada a relação das espécies coletadas, acompanhadas, sempre que possível, do nome vulgar regional e do "habitat". Todo o material aqui referido pode ser encontrado no herbário do INPA.

\section{Discussão E CONCLUSões}

Dentre o material coletado, as famílias melhores representadas são, sem dúvida, Mo- raceae, Chrysobalanaceae e Leguminosae, seguindo-se outras com menor número de espécies. Como a coleta procedeu-se aleatoriamente através dos "habitats", observa-se que há um equilíbrio entre floração e/ou frutificação da mata de terra firme e matas de igapós durante os períodos de coleta. A relação das espécies indica que o período de setembro a outubro parece ser o eleito para a floração e/ou frutificação de Moraceae e Chrysobalanaceae na região, evidentemente com exceções.

Observou-se, também, que algumas plantas típicas da floresta amazônica, como, por exemplo, Hevea brasiliensis, algumas espécies de Parkia e Cedrela não estão contidas na relação, porém outras representantes das famílias a que pertencem estas plantas estão

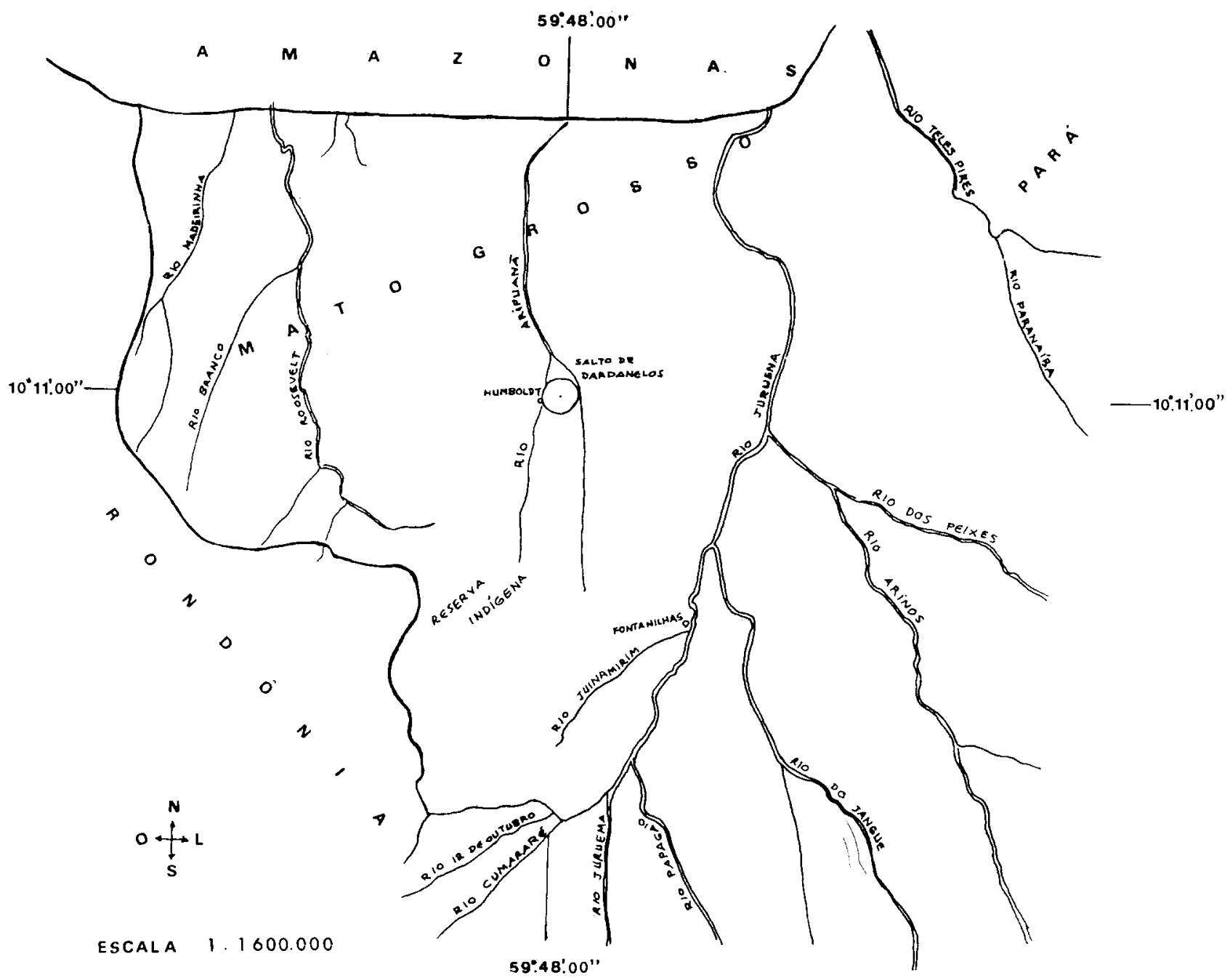

Fig. 1 - Localização geográfica da estação Humboldt em Aripuanã - MT (Mapa adaptado de Brasil. M. P. C. G., Projeto Aripuanã, 1973). 
presentes. As espécies citadas acima não estavam em processo de reprodução, porém suas presenças na área já foram assinaladas pelo Projeto Radam (Brasil. D.N.P.M., 1974).

O gráfico 1 é composto pelas famílias mais bem representadas na área, cujas espécies estavam com flor ou fruto. Vale ressaltar que, dentro de uma área de coleta, prati- camente todas as plantas férteis foram cole. tadas, daí acreditar-se que as percentagens dessas espécies contidas no gráfico 1 fornecem uma pequena indicação do que pode ser a fenologia local. As famílias mais bem representadas na área evidentemente apresentam maior possibilidade de conter espécies em estado reprodutivo.

\section{RELAÇAO DAS ESPÉCIES COLETADAS NO ARIPUANÃ (MT)}

Número de

Herbário

Nome vulgar

53.211

53.155

52.977

53.064

53.143

53.203

53.134

53.081

53.180

41.896

53. 209

53.066

41.983

41.834

41.926

53.150

53. 026

53.037

53.228

53.110

53.031

53.202

53.103

53.218

52.984

52.983

53.029

52.973

53.196

53.043
Envireira

Envira fofa

Janaguba

Titica

Grão de galo

Gravatá

Breu-de-leite

Breu

Breu-manga

Breu peludo

Breu almécega
Nome Gientífico

Habitat

\section{ANACARDIACEAE}

Anacardium sp.

\section{ANONACEAE}

Anona sp.

Duguetia flagellaris Huber

Duguetia spruceana R.E. Fr.

Guatteria discolor R.E. Fr.

\section{APOCYNACEAE}

Bonafusia tetrastachya (HBK) Mgf.

Himatanthus articulatus (Vahl) Woods.

ARACEAE

Anthurium gracile (Rudge) Engl.

Philodendron riedelianum Schott

BIGNONIACEAE

Arrabidaea fanshawei Sandw.

Clytostoma binatum (Thumb.) Sandw.

Cuspidaria laterifolia (Mart.) DC.

Cydista aequinoctialis (L.) Miers

Cydista lilacinea A. Gentry

Memora aff. patula Miers

Pleonotoma melioides (S. Moore) A. Gentry

BORRAGINACEAE

Cordia nodosa Lam.

Cordia silvestris Fresen

Heliotropium heliphillum Mart.

\section{BROMELIACEAE}

Araeococcus micranthus Brong.

Aechmea angustifolia $P$. \& $E$.

Aechmea cf. huebneri Harms

Aechmea aff. mertensii (Meyer) Schult.

Tillandsia bulbosa Hook.

BURSERACEAE

Paraprotium amazonicum Cuatr.

Protium plagiocarpium Benoist.

Tetragastris altissima (Aubl.) Swartz

Tetragastris unifoliolata (Engl.) Cuatr.

Tetragastris trifoliolata (Engl.) Cuatr.

CAPPARIDACEAE

Capparis $\mathrm{sp.}$
Igapó

Igapó

Terra firme

Terra firme

Terra firme

Igapó

Terra firme

Igapó

Terra firme

Terra firme

Igapó

Igapó

Igapó

Terra firme

Igapó

Igapó

Terra firme

Terra firme

Igapó

Terra firme

Terra firme

Terra firmo

Terra firme

Terra firme

Terra firme

Terra firme

Terra firmo

Terra firme

Terra firme

Terra firme 
Número de
Herbário

$\begin{array}{ll}\text { 53.019 } & \text { Piquiarana } \\ 41.831 & \text { Piquiarana do igapó }\end{array}$

53.192

41.606

53.230

53.095

52.980

53.157

53.161

41.612

53.235

41.603

43.412

53.144

53.045

53.238

43.424

53.067

53.233

41.982

41.801

4.1 .932

53.210

53.119 Maravilha

53.173

53.149

53.216

53.141

53.079

53.117

52.976

53.142

41.812

53.193

53.058

Caripé branco

Pajurazinho
Nome Científico

Habitat

CARYOCARACEAE

Caryocar glabrum Aubl.

Caryocar microcarpum Ducke

CELASTRACEAE

Maytenus sp.

Terra firme

Terra firme

Terra firme

\section{CHRYSOBALANACEAE}

Couepia paraensis (Mart. \& Zucc.) Benth. subsp. glaucescens (Spr. ex Hook. f.) Prance

Hirtella gracilipes (Hook. f.) Prance

Hirtella hispidula Mì

Hirtella racemosa Lam. var. racemosa

Licania blackii Prance

Licania canescens $R$. Ben.

Licania egleri Prance

Licania gardneri (Hook. f.) Fritsch

Licania heteromorpha Benth. var. heteromorpha

Licania cf. indurata Pilger

Licania kunthiana Hook. $f$.

Licania octandra (Hoffmgy. ex R. \& S.) subsp. pallida (Hook. f.) Prance

Licania parviflora Benth.

Licania parvifolia Hub.

Licania polita Spruce ex Hook. f.

Licania sclerophylla (Mart. ex Hook. f.) Fritsch.

Parinari excelsa Sabine

\section{COMPOSITAE}

Wulffia baccata $O$. Kuntze

Vernonia sp.

Capoeira

Igapó

\section{CONVOLVULACEAE}

Dicranostylis sp.

Igapó

\section{CYPERACEAE}

Hypolytrum longifolium L. C. Rich.

Igapó

DILLENIACEAE

Davilla kunthii St. Hil

Doliocarpus brevipedicellatus Garcke

Doliocarpus sagotianus Kubit.

\section{ELAEOCARPACEAE}

Sloanea $s p$.

\section{ERYTHROXYLACEAE}

Erythroxylum albertianum Kuhlm, \& W. Rodr.

Erythroxylum sp.

EUPHORBIACEAE

Actinostemon schomburgkii (Kl.) Hoch.

FLACOUR'TIACEAE

Casearia javitensis HBK

Lindackeria sp.

Capoeira

GESNERIACEAE

Drymonia coccinea (Aubl.) Wiehler

Terra firme

GNETACEAE

Gnetum sp.
Terra firme

Igapó

Terra firme

Terra firme

Terra firme

Terra firme

Igapó

Terra firme

Igapó 
Número de

Herbário

53.071

53.057

53.237

53.062

41.520

53.159 Pimenta de nambú

53.108

53.018

53.132

53.096

41.799

53.030

53.145

53.198

53.205

53.063

53.028

53.243

53.156

53.183

41.558

41.992

52.968

53.177

52.966

41.712

53.040

41.717

52.981

53.231

41.808

41.611

53.115

53.098

41.955

41.704

53.123

53.229

53.146

43.319

Nome vulgar

Louro aritú

Louro

Louro

Castanha-do-pará

Tauari

Castanharana

Rabo de camaleão

Ingá cipó

Ingá cipó

Ingá de macaco

Faveira

Sucupira preta

Sacupembinha

Jatobá

Arapari

Pau-santo

Cumarú

Tento preto

Mututi

Pau de rosas
Nome Gientífico

Habitat

GUTTIF'ERAE

Caraipa excelsa Ducke

Clusia weddeliana $\mathrm{Pl}$. \& $\mathrm{Tr}$.

Lorostemon sp.

Rheedia sp.

Terra firme

Terra firme

Igapó

Igapo

HUMIRIACEAE

Saccoglottis acaulis (Smith) Schltz.

LACISTEMACEAE

Lacistema aggregatum (Berg) Rusby

LAURACEAF

Anabi, Pau de cobra
Licaria aff. aurea (Hub.) Kosterm.

Ocotea cujumari Mart.

Ocotea opifera Meissn.

LECYTHIDACEAE

Bertholletia excelsa HB.

Cariniana domestica (Mart.) Miers

Couratari macrosperma A. C. Smith

Eschweilera fracta Kunth

LEGUMINOSAE MIMOSOIDEAE

Acacia paniculata Willd.

Inga edulis Mart.

Inga ingoides (Rich.) Willd.

Pithecolobium inaequale Bth.

Pithecolobium pedicellare Bth.

Pithecolobium sanguineum Bth.

LEGUMINOSAE CAESALPINIOIDEAE

Cassia adiantifolia $B$ th. var. pteridophylla (Sandw.) Ducke

Cassia fastuosa Willd.

Cassia lucens Vog.

Dialium sp.

Macrolobium acaciifolium (Benth.) Benth.

Macrolobium angustifolium (Benth.) Cowan

Swartzia bracteosa Ducke

Swartzia recurva Poeppig.

Zollernia paraensis Huber

LEGUMINOSAE PAPILIONOIDEAE

Dalbergia ecastophyllum (L.) Taub.

Dipteryx odorata (Aubl.) Wilid.

Ormosia paraensis Ducke

Pterocarpus amazonicus Hub.

\section{LOGANIACEAE}

Potalia amara Aubl.

Strychnos erichsonii Rich.

Strychnos guianensis (Aubl.) Mart.

Strychnos peckii B. L. Robinson

LYTHRACEAE

Cuphea sp.

Physocalymma scaberrimum Pohl

MALPIGHIACEAE

Heteropterys macrostachya Adr. Juss.
Hymenaea courbaril L.
Terra firme

Terra firme

Terra firme

Terra firme

Terra firme

Terra firme

Igapó

Terra firme

Terra firme

Terra firme

Igapo

Igapó

Igapó

Terra firme

Igapó

Terra firme

Igapó

Igapó

Igapó

Igapó

Igapó

Igapó

Igapó

Terra firme

Igapo

Igapo

Igapo

Terra firme

Igapo

Igapó

Igapó

Igapó

Terra firme

Igapo
Capoeira 
Número de

Herbário

53. 105

53.224

53.056

41.879

41.824

41.811

41.987

41.548

41.775

43.372

43.380

41.593

53.191

41.729

53.195

53.070

53.072

53.213

41.985

41.681

43. 288

53.131

41.330

41.826

41.782

41.775

43.335

41.731

43.336

43.301

41.828

43.323

41.757

41.711

41.877

42.033

41.989

41.980

41.745

41.695

41.779

41.702

53.027

41.917

53.036

43.300

41.967
Nome vulgar

Nome Científico

Habitat

MALVACEAE

Hibîscuss aff. dimidiatus Schrank

Igapó

MARCGRAVIACEAE

Marcgravia parviflora Rich. Igapó

Souroubea guianensis Aubl.

Igapó

MELASTOMATACEAE

Aciotis indecora (DC.) Triana var. macrophylla Cogn.

Miconia affins DC.

Miconia chrysophylla (Rich.) Urb.

Buxixú

Fruto de jacu

Buxixú de formiga

Buxixú de formiga

Buxixú de formiga

Miconia ibaguensis (Bonpl.) Triana

Miconia nervosa (Sw.) Triana

Miconia prasina (Sw.) DC.

Miconia punctata (Ders.) Don.

Miconia cf. regelii $\operatorname{Cog}$.

Miconia tiliifolia Nauc.

Mouriri sagotiana Triana

Tococa egensis Naud.

Tococa guianensis Aubi.

Tococa longisepala Cogn.

MELIACEAE

Trichilia brachystachya $\mathrm{Kl}$. Igapó

Trichilia sp.

\section{MENISPERMACEAE}

Cipó abuta

Cipó quina

Pau rainha

Gameleira roxa

Apui

Apui

Apui

Inharé

Inharé
Abuta grisebachii Tr. \& Planch

Anomospermum bolivianum Krukoff \& Moldenke

Orthomene schomburgkii (Miers) Barneby \& Krukoff MONIMIACEAE

Siparuna guianensis Aubl.

\section{MORACEAF}

Bagassa guianensis Aubl.

Batocarpus amazonicus (Ducke) Fosberg

Brosimum guianensis (Aubl.) Huber

Brosimum lactescens (S. Moore) C.C. Berg

Brosimum rubescens Taub.

Brosimum utile (HBK) Pittier subsp. ovatifolium

Castilla ulei Warb.

Cecropia concolor Wild.

Cecropia ficifolia Sneíhl.

Cecropia riparia Snetil.

Coussapoa araneosa Standl.

Coussapoa trinervia Spr. ex Mildbr.

Dorstenia asaroides Gard.

Ficus frondosa Stand!

Ficus gomelleira Kunth \& Bouche

Ficus mathewsii (Miq.) Miq.

Ficus nymphaeifolia P. Mill.

Ficus paraensis (Miq.) Miq.

Ficus pertusa $L$. $f$.

Ficus trigona L. f.

Helianthostylis sprucei Baill.

Helicostylis, elegans (Macbr.) C. C. Berg

Helicostylus pedunculata $R$. Ben.

Helicostylis tomentosa (P. \& E.) Rusby

Maquira coriacea (Karsten) C. C. Berg
Terra firme

Capoeira

Capoeira

Igapó

Terra firme

Terra firme

Terra firme

Terra firme

Igapó

Terra firme

Igapó

Igapó

Igapó

Igapó

Igapó

Terra firme

Igapó

Terra firme

Terra firme

Terra firme

Terra firme

Terra firme

Igapó

Igapó

Terra firme

Capoeira

Capoeira

Igapó

Terra firme

Igapó

Terra firme

Terra firme

Igapó

Igapó

Terra firme

Terra firme

Terra firme

Terra firme

Terra firme

Terra firme

Terra firme

Terra firme

Terra firme 
Número de
Herbário

41.913

41.820

41.546

41.837

43.291

43.365

41.827

43.362

41.555

41.509

41.915

41.680

41.678

43.348

43.350

41.829

41.819

41.919

41.524

41.840

43. 371

43.351

41.547

41.832

41.900

41.848

53.069

$\begin{array}{ll}53.154 & \text { Murta } \\ 53.165 & \text { Pedra ume-caá } \\ 53.163 & \text { Ubaia } \\ 53.136 & \text { Murta grande }\end{array}$

53.039

52.974

52.972

53.118

53.167

53.112

53.086

52.964

53. 189

53.124

53.128

53.129

53.099

Nome vulgar
Nome Gientifico

Maquira guianensis Aubl.

Nauclecpsis caloneura (Huber) Ducke

Naucleopsis glabra Spr. ex Baillon

Naucleopsis macrophylla Miq.

Naucleopsis stipularis Ducke

Naucleopsis ternstroemiiflora (Mildbr.) C. C. Berg

Perebea molis (P. \& E.) Huber subsp. mollis

Pourouma asuminata Mart.

Pourouma guianensis Aubl.

Pourouma minor $R$. Benoist

Pseudolmedia laevigata Tréc.

Pseudolmedia laevis (R. \& P.) Macbr.

Pseudolmedia macrophylla Tréc.

Psendolmedia murure Standl.

Pseudolmedia rigida ( $\mathrm{Kl}$. \& Kers.) C. C. Berg subsp rigida

Sorocea guilleminiana Gaud.

Sorocea muriculata Miq.

MUSACEAE

Heliconia sp

\section{MYRISTICACEAE}

Campsoneura ulei Warb.

Iryanthera juruensis Warb.

Iryanthera laevis Mkgf.

Iryanthera paraensis Hub.

Iryanthera ulei Warb.

Virola calophylla Warb.

Virola clongata (Benth.) Warb.

Virola pavonis (A.DC.) A.C. Smitn

\section{MYRSINACEAE}

Conomorpha sp.

MYRTACEAE

Eugenia biflora DC.

Eugenia coarensis DC.

Eugenia aff. subterminalis DC.

Myrcia fallax (Rich.) DC.

\section{NYCTAGINACEAE}

Neea aff. mollis Spruce

Neea aff. ovalifolia Spruce

CCHNACEAE

Ouratea aff. chrysopetala Ule

Ouratea paraensis Huber

Erva de S. Martinho

Muiragiboia preta

Sauvagesia erecta Linn.

\section{OLACACEAE}

Aptandra spruceana Miers

\section{ORCHIDACEAE}

Brassavola martiana Lindl.

Encyclia vespa (Vell.) Dress.

Zygosepalum labiosum (L. C. Rich.) Gnray

PALMAE

Astrocaryum munbaca Mart.

Euterpe aff. controversa Barb. Rodr.

Euterpe precatoria Mart.

Oenocarpus minor Mart.
Habitat

Terra firme

Capoeira

Terra firme

Terra firme

Igapó

Terra firme

Capoeira

Terra firme

Terra firme

Terra firme

Terra firme

Terra firme

Terra firme

Igapó

Igapó

Terra firme

Terra firme

Terra firme

Terra firme

Igapó

Terra firme

Igapó

Terra firme

Terra firme

Terra firme

Terra firme

Igapó

Igapó

Igapó

Igapó

Terra firme

Terra firme

Terra firme

Terra firme

Igapó

Igapó

Terra firme

Igapó

Igapó

Várzea

Terra firme

Terra firme

Terra firme

Terra firme 
Número de

Herbário

43.425

53.201

53.242

53.236

53.179

53.223

53. 093

53.038

52.963

52.971

53.055

Saca rolha

53.059

53.094 Pente de macaco

53.227

53.240

$53.068 \quad$ Viuvinha

53. 182

53.078
Nome Científico

Habitat

\section{PIPERACEAE}

Peperomia sp.

Terra firme

POLYGONACEAE

Coccoloba racemulosa Meiss. Igapó

PROTEACEAE

Panopsis rubescens (Pohl) Pittier Igapó

Roupala obtusata Klotzch Igapó

QUIINACEAE

Quiina paraensis Pires \& Fróes

Nilna oppositifolia (Pires) Pires

RUTACEAE

Zanthoxyitim sp. SAPINDACEAE

Cupania hirsuta Radlk.

Matayba purgans (P. \& E.) Radlk. SAPOTACEAE

Pouteria aff. gutta (Ducke) Baehni STERCULIACEAE

Helicteris aff. guazumiifolia HBK. STYRACACEAE

Styrax guianensis A. DC. TILIACEAE

Apeiba tibourbou Aubl. UMBELIFERAE

Eryngium aff. ebracteatum Lam. Igapó VITACEAE

Cissus aff. gongylodes Burch. ex Bak. Igapó VERBENACEAE

Petraea bracteata Standl. ZINGIBERACEAE

Costus scaber R. \& P. Renealmia aff. floribunda $\mathrm{K}$. Sch.
Terra firme Igapó

Terra firme

Terra firme

Terra firme

Terra firme

Terra firme

Terra firme

Terra firme

Terra firme

Igapó

Igapó

\section{AgradeCimentos}

Aos botânicos especialistas que nos ajudaram na identificação do material coletado: Drs. A. Gentry, C. C. Berg, Murça Pires, P. I. Braga, T. Koyama, W. Rodrigues. Aos auxiliares José Ramos, Osmarino Monteiro, Dionísio Coelho e Geraldo Mota, que nos ajudaram na coleta e identificação das plantas.

\section{SUMMARY}

A list of species from Aripuanã (MT) flora is given by the authors. The material listed is only of the fertile plants collected belonging to the groups of Gymnospermae and Angiospermae. They were collected during the months October (1973) and September and October (1975). The families with most number of species in flower or fruit in September ond October were Moraceae, Leguminosae and Chrysobalanaceae.

\section{BIBLIOGRAFIA CITADA}

Brasil. D.N.P.M., Projeto Radam

1974 - Estudos e levantamentos de recursos naturais. Area Programa Aripuanã. Relatório final.

Brasil. M.P.C.G., Projeto Aripuañ̃

1973 - Projeto Aripuanã - Instituto de Planejamento Econômico e Social (IPEA), 96 p., 6 Mapas.

PIRES, J.M.

1973 - Tipos de vegetação da Amazônia. Mus. Par. Emilio Goeldi. Publicaçōes avulsas, $20: 179-202$. 